\title{
Comparison of in Hospital Outcome of Patients with and without Distorted Terminal Portion of QRS Complex on Initial Electrocardiogram in ST Segment Elevation Myocardial Infarction
}

KHANDAKER AISHA SIDDIKA ${ }^{1}$, MD. ABU SIDDIQUE ${ }^{1}$, SHAMIM AHSAN ${ }^{1}$, ARIF HOSSAIN ${ }^{1}$, SOHEL MAHMUD ${ }^{1}$, HIRANMOY DAS ${ }^{1}$, MOHAMMAD ZAKIR HOSSAIN ${ }^{1}$, DIPAL K. ADHIKARY ${ }^{1}$, ARIF MOHAMMAD SOHAN ${ }^{1}$, HARISUL HOQUE ${ }^{1}$

${ }^{1}$ Department of Cardiology, Bangabandhu Sheikh Mujib Medical University, Dhaka

Address of Correspondence: Dr. Khandaker Aisha Siddika, Department of Cardiology, Bangabandhu Sheikh Mujib Medical University, Dhaka, e-mail: dr.aishajahangir.bsmmu@gmail.com

\begin{abstract}
:
Background: Distorted terminal portion of QRS complex on initial electrocardiogram in ST segment elevation myocardial infarction is a strong predictor of inhospital adverse outcome. Objectives: Our purpose of this study was to analyse admission ECG in patients of STEMI based on terminal portion of QRS complex and find out inhospital death, heart failure, cardiogenic shock and recurrent MI. Methods: We evaluated 60 patients of STEMI admitted within 12 hours and receiving thrombolytic therapy. We defined two ECG groups according to absence of distortion of terminal QRS (Group-I) and presence of distorted terminal QRS (Group-II) in two or more adjacent leads. Group-II further divided into pattern-A - J point originating at $\geq 50 \%$ of height of $R$ wave in leads with $q R$ configuration and pattern $B$ - $S$ wave is absent in leads with $R S$ configuration. Results: Out of 60 patients of STEMI, 30(50.0\%) patients had distortion of QRS. There were 7(11.6\%) deaths, 16(26.7\%) heart failure, 3(5.0\%) cardigenic shock and no recurrent myocardial infarction. Hospital mortality and heart failure were found to be significantly higher in distorted QRS group (3.3\% vs. $20.0 \%, p=0.04 ; 13.3 \%$ vs. $40.0 \%, p=0.02$; respectively), cardiogenic shock of both groups did not show significant difference ( $0.0 \%$ vs. $10.0 \%, p=0.075)$. Multiple logistic regression analysis using hospital mortality as dependable variable and all studied risk factors were independent variables, QRS distortion on admission ECG and Killip class were only variable found to be statistically significant $(O R=7.25$, p value $<0.05 ; O R=16.25$, $p$ value $<0.05$ respectively). Conclusion: Careful analysis of ECG which is simple, cheap, universally available bed side investigation may offer important prognostic information in patients with STEMI and would help in deciding which patients should go urgent myocardial revascularization procedure.
\end{abstract}

Key words: STEMI, Distorted QRS, Death, Heart failure, Cardiogenic shock.

Introduction:

Ischaemic heart diseases is a global health burden including Bangladesh and one of the major cause of mortality and morbidity. In a study of WHO non communicable diseases country profile, 2014 showed $17 \%$ death was due to cardiovascular diseases ${ }^{1}$. For a long time the 12-lead electrocardiogram (ECG) is considered an essential part of the diagnosis and initial evaluation of patients with chest pain ${ }^{2}$. ECG reflects the physiology of the myocardium during acute ischaemia, where as the coronary angiography identifies vessel anatomy ${ }^{3}$. For this reason, coronary angiography remains the "gold standard" for identifying the infarct related artery and the ECG remains the gold standard for identifying the presence and location of acute myocardial infarction ${ }^{3}$. Patients presenting with STEMI requiring risk assessment shortly after admission, when only history, physical examination and the ECG are available ${ }^{4}$. The purpose of this study was to analyse admission ECG in patients of STEMI based on terminal portion of QRS complex and find out inhospital death, heart failure, cardiogenic shock and recurrent MI. This study provide information regarding prognostic value of interpretation of ECG. With this cost effective and easily available diagnostic tool, we can easily predict the outcome of patient and help in early referral, follow up and decision regarding early myocardial revascularization in patient with STEMI. Thus improve overall approach of management of the patients and prevent early cardiac death.

Methods:

Patients: This observational prospective study was conducted in BSMMU during the period of July 2014 to June 2015. We studied 60 patients of STEMI admitted within 12 hours of onset of chest pain which lasted for at 
least 30 minutes and receiving thrombolytic therapy. STEMI was diagnosed when ECG showing ST elevation of $1 \mathrm{~mm}$ or more in two or more consecutive leads often with reciprocal ST depression in the contralateral leads, except in leads V2-V3 where $\geq 2 \mathrm{~mm}$ of ST elevation in men with age $\geq 40$ years, $\geq 2.5 \mathrm{~mm}$ men with age $<40$ years and $\geq 1.5 \mathrm{~mm}$ in women were required for accurate diagnosis ${ }^{5}$ with positive $\mathrm{T}$ waves in leads with ST segment elevation with increase in cardiac enzymes. Patients with admitted after 12 hours of STEMI and who had absolute contraindication of Streptokinase, left bundle branch block (LBBB), ventricular arrhythmias (Ventricular tachycardia, Ventricular fibrillation) at admission, ventricular pacing or negative $\mathrm{T}$ waves in leads demonstrating ST segment elevation were excluded ${ }^{4}$. Clinical assessment was done and various risk factors were noted. Standard medication were received as per the direction of the treating physician.

ECG analysis: Terminal portion of the QRS complex in two or more consecutive leads were analysed by 3 experienced physicians and in case of uncertain ECG classification, two other physicians were consulted. The investigators analyzing ECGs were blinded to clinical data of the patients. Anterior localization and number of leads with ST segment elevation were analyzed. The patients were classified into two ECG pattern groups, group I and group II. Group II was divided into A \& B pattern.

Group I: Those with tall symmetric $\mathrm{T}$ waves and ST elevation in two or more adjacent leads without major changes in the configuration of the terminal portion of the QRS complex (Fig. 1 and Fig. 2).

Group II: Those with tall symmetric $\mathrm{T}$ waves and ST elevation and distortion of the terminal portion of the QRS complex in two or more adjacent leads.

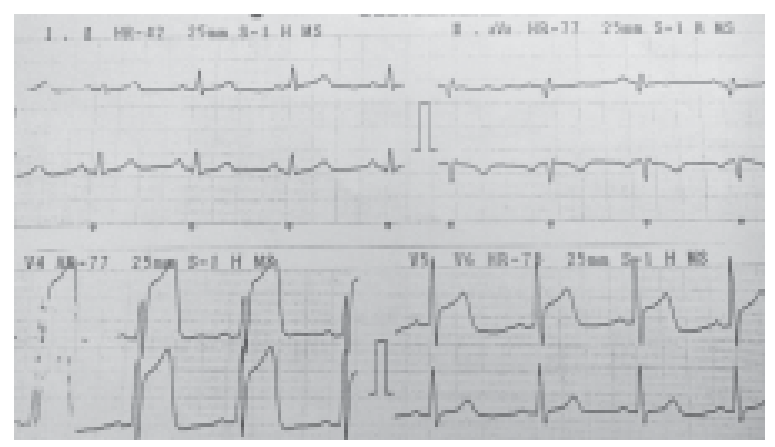

Fig.-1: Mr. X, 50 years admitted in BSMMU in CCU-2 on 22.03.2015 with the complaints of severe chest pain and ECG at admission showing acute STEMI anterior. There is presence of S wave in leads V3, V4 and V5 in Leads with Rs configuration, without distortion of the QRS.

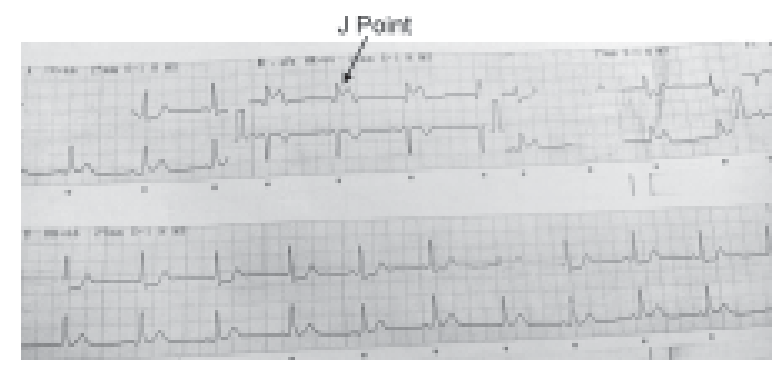

Fig.-2: Mr. X, 77 years admitted in BSMMU in CCU-2 on 28.02.2015 with the complaints of severe chest pain for 1 hour and ECG at admission showing acute STEMI inferior, $J$ point emergence below $50 \%$ of the $R$ wave amplitude, without distortion of the QRS.

Pattern A: The height of the $\mathrm{R}$ wave and $\mathrm{J}$ point were measured. Emergence of the J point at or above the lower half of the $\mathrm{R}$ wave ( $\geq 50 \%$ of the $\mathrm{R}$ wave amplitude as measured from isoelectric line) in leads with qR configuration (Fig. 3 and Fig. 4).

Pattern B: Absence of $\mathrm{S}$ waves in leads with Rs configuration (leads without Q waves) (Fig. 5)

Outcomes: The analysis of the inhospital clinical course including the occurrence of death, heart failure, cardigenic shock and recurrent MI. Heart failure was assessed by Killip class classification.

Statistical analysis: Data was analysed by using statistical package for social sciences (SPSS) version 20. Qualitative data expressed as percentage and difference between two groups were analysed by Chi-square test. Quantitative data expressed as mean \pm SD and difference between two groups were analysed by unpaired two tailed t test.

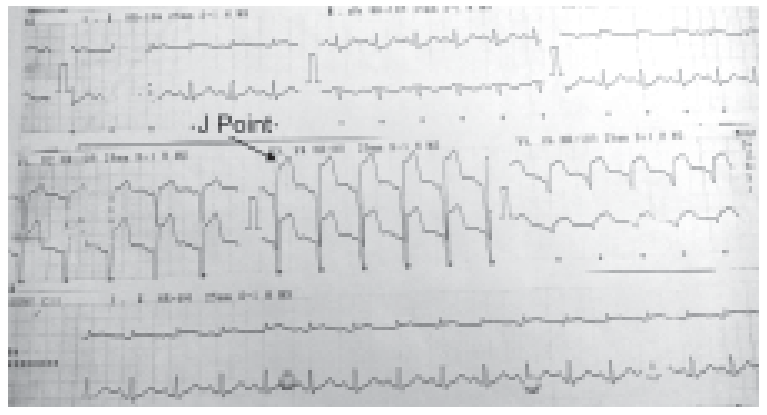

Fig.-3: $M r . X$, 45 years admitted in BSMMU in CCU -2 on 20.02.2015 with the complaints of severe chest pain for 10 hour and ECG at admission showing acute STEMI extensive anterior. J point emergence more than $50 \%$ of the $R$ wave amplitude in leads V1-V6, 1 and aVL in Leads with $q R$ configuration, pattern A. 


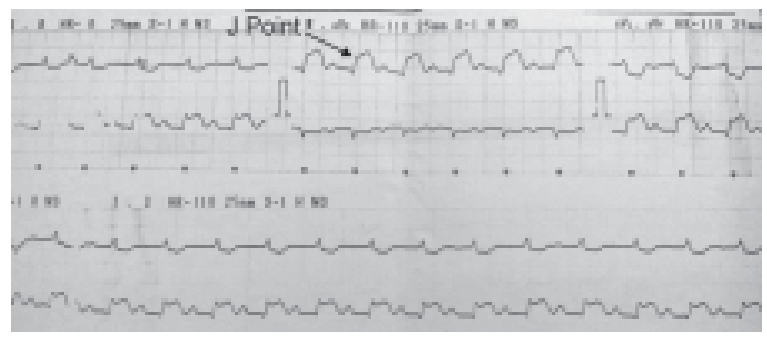

Fig.-4: Mr. X, 62 years admitted in BSMMU in CCU -2 on 13.02.2015 with the complaints of severe chest pain and breathlessness for 8 hours and ECG at admission showing acute STEMI inferior with fast degree AV block. J point emergence more than $50 \%$ of the $R$ wave amplitude, in leads II, III and aVF in Leads with qR configuration, pattern $A$.

Multiple logistic regression analysis were done to exclude confounding variables for adverse events. Odds ratio was analysed to assess the risk of imposed QRS distortion for adverse cardiac events. p value $<0.05$ was considered for statistically significant.

\section{Results:}

A total number of 60 patients (30 patients in each group) with STEMI, out of them 55 patients were male and mean age was 55 years, 54.33 \pm 10.37 in Group I and 57.03 \pm 11.91 in Group II. There were no statistically significant difference in risk factors and door to needle time in between two groups (Table-1). Regarding inhospital

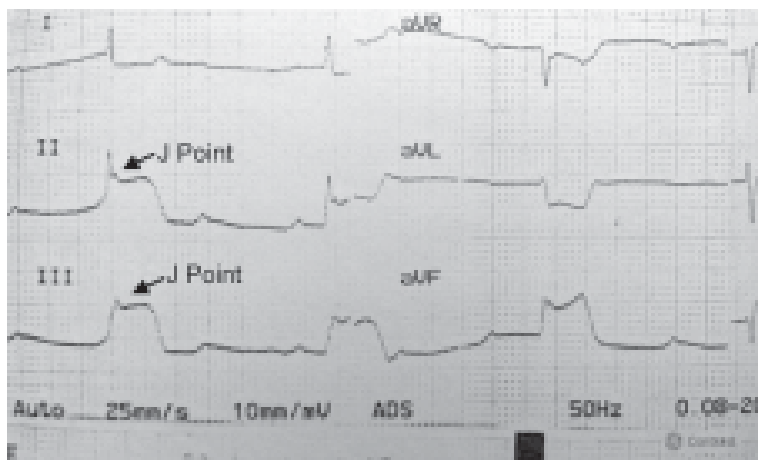

Fig.-5: Mr. $X$, 55 years admitted in BSMMU in CCU -2 on 22.01.2015 with the complaints of severe chest pain for 3 hours and ECG at admission showing acute STEMI inferior and complete heart block. Absence of S wave in leads II, III and aVF in Leads with Rs configuration, pattern B.

outcome, death and heart failure were significantly higher in group II (3.3\% vs. 20.0\%, p=0.04; 13.3\% vs. 40.0\%, $\mathrm{p}=0.02$; respectively), cardiogenic shock of both groups did not show significant difference $(0.0 \%$ vs. $10.0 \%$, $\mathrm{p}=0.075$ ) but there was no recurrent $\mathrm{MI}$ in two groups (Fig. 6). Among study population 7 patients died due to cardiogenic shock (2), acute left ventricular failure (2) and arrhythmias (3). Out of 60 patients Killip $\geq 2$ were 16 patients, 2 died from distorted QRS group ( $\mathrm{p}<0.05$ ). Multiple logistic regression analysis showed that the distorted QRS on initial ECG and Killip class (e”II) were independent predictor of heart failure and inhospital mortality (Table-2).

Table-I

Showing characteristics of 60 patients of STEMI without QRS distortion (Group I) and QRS distortion (Group II) on admission ECG.

\begin{tabular}{lccc}
\hline Characteristics & Group-I $(\mathrm{n}=30)$ & Group-II $(\mathrm{n}=30)$ & p value \\
\hline Age (mean) & $54.33 \pm 10.37$ & $57.03 \pm 11.91$ & $0.353^{\mathrm{ns}}$ \\
Sex (Male) & $28(93.3 \%)$ & $27(90.0 \%)$ & $0.640^{\mathrm{ns}}$ \\
HTN & $9(63.3 \%)$ & $13(43.3 \%)$ & $0.121^{\mathrm{ns}}$ \\
DM & $14(46.7 \%)$ & $20(66.7 \%)$ & $0.118^{\mathrm{ns}}$ \\
Smoking & $16(53.3 \%)$ & $17(56.7 \%)$ & $0.795^{\mathrm{ns}}$ \\
Dyslipidemia & $13(43.3 \%)$ & $15(50.0 \%)$ & $0.604^{\mathrm{ns}}$ \\
Time to thrombolysis (mean \pm SD) hrs & $3.9 \pm 1.13$ & $4.5 \pm 1.27$ & $0.06^{\mathrm{ns}}$ \\
Hear rate (e”100/min) & $4(13.3 \%)$ & $8(26.7 \%)$ & $0.795^{\mathrm{ns}}$ \\
Killip class (e” II) & $4(13.3 \%)$ & $12(46.7 \%)$ & $0.019^{*}$ \\
Location of AMI (Anterior) & $15(50.0 \%)$ & $16(53.3 \%)$ & $0.796^{\mathrm{ns}}$ \\
Number of leads with ST segment elevation $(\geq 5)$ & $13(43.3 \%)$ & $16(53.3 \%)$ & $0.438^{\mathrm{ns}}$ \\
\hline
\end{tabular}


Table-II

Showing multiple logistic regression analysis using hospital mortality as dependable variables $(n=60)$.

\begin{tabular}{|c|c|c|c|c|}
\hline \multirow[t]{2}{*}{ Variables } & \multirow[t]{2}{*}{ Odds ratio } & \multicolumn{2}{|c|}{$95 \%$ CI } & \multirow[t]{2}{*}{ p value } \\
\hline & & Lower & Upper & \\
\hline Age: $<60$ vs $\geq 60$ & 0.267 & 0.038 & 1.85 & 0.181 \\
\hline Sex: Male vs Female & 2.143 & - & - & 0.96 \\
\hline HTN: Yes/No & 2.857 & 0.415 & 19.65 & 0.286 \\
\hline DM: Ys/No & 1.500 & 0.238 & 9.465 & 0.666 \\
\hline Smoking: Yes/No & 0.333 & 0.31 & 3.579 & 0.364 \\
\hline Time to thrombolysis $\geq 4$ hrs: Yes vs No & 3.750 & 0.540 & 26.05 & 0.181 \\
\hline Hear rate: $\geq 100 \mathrm{vs}<10 \overline{0}$ & 1.600 & 0.202 & 12.69 & 0.656 \\
\hline Killip class: $\geq$ II vs I & 16.25 & 1.774 & 148.84 & $0.006^{*}$ \\
\hline Location of AMI: Ant. vs Inf. & 5.00 & 0.704 & 35.49 & 0.108 \\
\hline$\geq 5$ leads with ST elevation: Yes vs No & 0.667 & 0.106 & 4.21 & 0.666 \\
\hline QRS distortion: Yes/No & 7.25 & 0.76 & 171.25 & $0.04 *$ \\
\hline
\end{tabular}

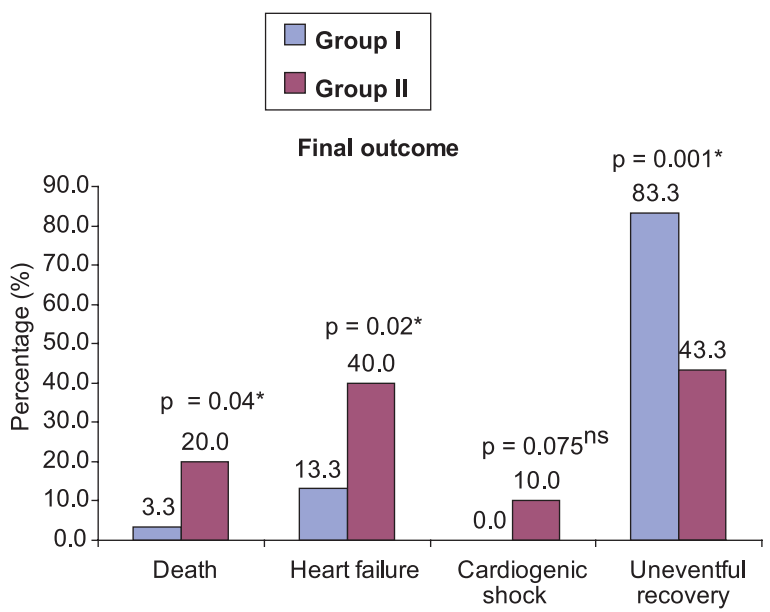

Fig.-6: Percentage of patients developed death, heart failure, cardiogenic shock and uneventful recovery $(n=60)$

\section{Discussion:}

Coronary artery disease (CAD) is an increasingly important medical and public health problem, and is the leading cause of mortality in Bangladesh. Like other South Asians, Bangladeshis are unduly prone to develop CAD, which is often premature in onset, follows a rapidly progressive course. An ECG, a source of decisive data that may obtain within 10 minutes after arrival of patients with a history of chest discomfort consistent with acute coronary syndrome ${ }^{6}$. The role of ECG in diagnosis and prognostication of acute myocardial infarction is well established. In acute MI who might benefit from reperfusion therapy either by mechanical or pharmacological can be identified with this cost effective, easily available and bedside diagnostic tool. Therefore, this study tried to determine the inhospital death, heart failure, cardiogenic shock and recurrent MI by interpretating the ECG and comparing the patients with STEMI with or without distorted terminal portion of QRS complex in ECG on admission.

The extent of myocardial infarction can also be estimated by Echocardiography, radionuclide ventriculography, myocardial perfusion scintigraphy, and magnetic resonance imaging (MRI). Positron emission tomography and X-Ray computed tomography (CT) are less common ${ }^{7}$. But the imaging methods such as Echo and radionuclide ventriculography increases delay in starting treatment and can neither measure the "severity" of ischemia nor differentiate between viable myocardium and already infracted myocardium ${ }^{4}$. Although single photon emission computed tomography (SPECT) with 99 mTc sestamibi can quantify residual flow to infarct area and the size of the area at risk and allow the viable myocytes to be imaged but this methods are also time consuming, expensive, technically demanding and not easily available ${ }^{4,7}$.

The purkinji system is less sensitive to ischemia than the contracting myocardial cells. Distortion in the terminal portion of the QRS to occur when more severe degree of ischemia that would injure the purkinji system ${ }^{8,9}$. Distortion of the QRS is caused by alteration in the conduction velocity of the activation wave in the purkinji fibers as it travels through the ischemic region. The delayed conduction decreases the degree of cancellation and, by so doing, increase the amplitude of the $\mathrm{R}$ wave and causes loss of the $\mathrm{S}$ wave. ${ }^{8,9}$ 
The mean door to needle time was $3.9 \pm 1.13$ hrs and $4.5 \pm 1.27$ hrs in group I and group II respectively. These parameter did not show statistically significant difference between two groups ( $\mathrm{P}$ value $>0.05$ for these parameters) that also similar to other studies $4,10,11,12$. Time interval between onset of chest pain and thrombolysis is an important factor for outcome of the patients ${ }^{13}$.

Birnbaum et al. ${ }^{8}$ found time interval to be significant when thrombolysis to be performed $\geq 2$ hrs and was associated with increase inhospital mortality only among patients with QRS distortion and not in patients without QRS distortion, patients who were treated within 2 hrs of onset of symptoms there was no difference in mortality between two groups ${ }^{14}$.

Our patient's Killip class $\geq$ II 13.3\% and 46.7\% respectively in group I and group II. These parameters showed the higher Killip class in group II (p value $<0.05$ ) which was observed by various study 4,8,11,14. They stated that higher the Killip class indicate larger area of myocardial necrosis and higher incidence of heart failure and increase mortality.

Regarding the ECG findings of these patients population only the QRS distortion were significant, location of anterior MI in group I and group II did not showed any significant difference. In present study ST segment elevation in $\geq 5$ leads in group I and group II $43.3 \%$ and $53.3 \%$ respectively ( $p$ value $>0.05$ for these parameters). These findings agreed with other study ${ }^{4,14}$. Terminal portion of QRS distortion on admission was a better predictor of final infarct size than number ST segment elevation. Patients with 8 or 9 of out of 12 leads showing ST segment elevation have three or four times of mortality of those with only 2 or 3 leads demonstrating ST segment elevation ${ }^{15}$. Birnbaum ${ }^{16}$ stated that the number of leads with ST elevation and sum of the ST elevation had only mild correlation with myocardial dysfunction but no correlation with severity of dysfunction. Severity correlate with the degree of distorted QRS complex.

Regarding final outcome of the study population, death was 3.3\% and 20\% respectively in group I and group II. Heart failure was 13.3\% without QRS distortion group and $40.0 \%$ in distorted QRS group. These parameters showed difference were significant between two groups ( $\mathrm{P}$ value $<$ 0.05 for these parameters) and similar pattern had also been found among many other studies $4,8,11,14$. Patients with distorted QRS complex had absence of collateral blood flow, larger area of myocardial necrosis and difficult to salvage the jeopardized myocardium even after reperfusion therapy. So this group of patients had more tendency to develop heart failure and death.

Among study population 7 patients died due to cardiogenic shock (2), acute left ventricular failure (2) and arrhythmias (3).

In multivariate analysis where hospital mortality was dependent variables and all the other baseline characteristics such as age, gender, HTN, DM, smoking, time to thrombolysis, heart rate, Killip class, location of acute MI, $\geq 5$ leads with ST elevation, initial ECG pattern were independent variables where Killip class and initial ECG pattern were only significantly associated with hospital mortality (P value $>0.05$ ). The odd ratio were more than one in gender, HTN, DM, time to thrombolysis, heart rate, location of acute MI. Results of current study had similarity with other study $4,11,12$. It indicates that the distorted QRS on ECG and Killip class were independent predictor of inhospital mortality and heart failure.

The morphology of QRS suggest the grade of ischemia, absence of distorted QRS indicate grade 2 ischemia and presence of distortion of QRS complex in acute MI indicate grade 3 ischemia that was predictive of more extensive myocardial involvement and greater severity of regional dysfunction $^{17}$.

\section{Conclusion:}

Careful analysis of ECG which is simple, cheap, universally available, bed side investigation may offer important prognostic information in patients with STEMI. The distortion of the terminal portion of QRS appears as an independent predictor for development of death and heart failure. That would help in deciding which patients should go for early aggressive myocardial revascularization procedures or urgent referral to higher centers even if much time has elapsed from onset of symptom.

\section{Limitations}

Although the result of this study supports the hypothesis there are some facts to be considered which might affect the results: Results were not correlated with coronary angiographic findings. Further required long term follow up for death, heart failure, cardiogenic shock and recurrent MI.

\section{Reference:}

1. WHO non communicable diseases counter profile, 2014.

2. Sclarovsky, S. Electrocardiography of acute myocardial ischaemic syndromes, London: Martin Dunitz, 1999.

3. Birnbaum, Y \& Deew, BJ. The electrocardiogram in ST elevation acute myocardial infarction: correlation with coronary anatomy and prognosis. Postgrad Med J, 2003; 79: 490-504. 
4. Mulay, DV \& Mukhedkar, SM. Prognostic significance of the distortion of terminal portion of QRS complex on admission electrocardiogram in ST segment elevation myocardial infarction. Indian heart journal, 2013; 65: 671-77.

5. Steg PG, James SK, Atar D et al. ESC Guidelines for the management of acute myocardial infarction in patients presenting with ST-segment elevation, 2013.

6. Sabatine, MS \& Cannon, CP. Approach to The Patient with Chest Pain. In: Mann, Dl, Zipes, DP, Libby, P (eds), Bonow, Ro, Braunwald's heart disease, A text book of cardiovascular medicine, 10th edn, Elsevier, Phyladelphia; 2015: 1057-67.

7. Kim, Mc, Kini, AS \& Fuster, V. Definitions of Acute Coronary Syndromes. In: Fuster, V, Walsh, R \& Harringlon, RA (eds), Hurst's The Heart, $13^{\text {th }}$ edn, McGraw Hill, New York, 2011; 1287-95.

8. Birnbaum, Y, Herz, I \& Sclarovsky, S, Zlotikamien B, Chetrit A, Olmpr L, Barbash, Gi. Prognostic significance of the admission electrocardiogram in acute myocardial infarction. J Am Coll Cardiol, 1996; 27(5): 1128-32.

9. Yang, HS, Lee, CW, Hong, MK, Moon, DH, Kim, YH, Lee, SG, Han, KH, Kim, JJ, Park, SW, Park, SJ. Terminal QRS complex distortion on the admission electrocardiogram in anterior acute myocardial infarction and association with residual flow and infarct size after primary angioplasty. The Koren Journal of Internal Medicine, 2005; 20: 21-25.

10. Birnbaum, Y, Maynard, C, Wolfe, S, Mager, A, Strasberg, B, Rechavia, E, Gates, K, Wagner, GS. Terminal QRS distortion on admission is better than ST segment measurement in predicting final infarct size and assessing the potential effect of thrombolytic therapy in anterior wall acute myocardial infarction. Am J Cardiol, 1999; 84: 530-34.
11. Garcia-Rubira, JC, Borbolla, RG, Gil, IN, Manzalo, NC, Romero, MAG, Ortiz, AF, Perez de, IL, Macaya, C. Distortion of the terminal portion of the QRS is predictor of shock after primary percutaneous coronary intervention for acute myocardial infarction. International Journal of Cardiology, 2008; 130: 241-45.

12. Sucu, MM, Karadede, A, Aydinalp, O, Ozturk, O, Toprak, N. The relationship between terminal QRS complex distortion and early low dose dobutamine stress echocardiography in acute anterior myocardial infarction. Japan heart Journal, 2003; 45: 373-86.

13. Jose VJ, Gupta SN. Mortality and morbidity of acute ST segment elevation myocardial infarction in the current era. Indian Heart J, 2004; 56: 210-14.

14. Garcia-Rubira, JC, Gil, IN, Borbolla, RG, Lennie, V, Manzano, NC, Cobos, MA, Perez de, IL, Ortiz, AF, Macaya, C. Distortion of the QRS in elderly patients with myocardial infarction, Cardiology journal, 2009; 16(5): 1-xxx.

15. Scirica, BM \& Morrow, DA. ST-Elevation Myocardial Infarction: Pathology, Pathophysiology, and Cilinical Features. In: Mann, Dl, Zipes, DP, Libby, P (eds), Bonow, Ro, Braunwald's heart disease, A text book of cardiovascular medicine, $10^{\text {th }}$ edn, Elsevier, Phyladelphia, 2015; 1068-93.

16. Birnbaum, Y, Crigerda, DA, Wagner, GS, Strasberg, B, Mager, A, Gates, K, Granger, CB, Ross, AM, Barbash, GI. Prediction of extent and severity of left ventricular dysfunction in anterior acute myocardial infarction by the admission electrocardiogram. Am Heart J, 2001; 141: 915-24.

17. Luna AB, Goldwasser D, Fiol M et al. Surface echocardiography. In: Fuster, V, Walsh, R \& Harringlon, RA (eds), Hurst's The Heart, $13^{\text {th }}$ edn, McGraw Hill, New York, 2011; 343. 